\title{
Study of the influence of group fitness training on the physical health of women aged 35-40
}

\author{
I. V. Lazunina ${ }^{1 *}$, and M. V. Kosheleva ${ }^{2}$ \\ ${ }^{1}$ Department "Adaptive Physical Culture, Sports and Tourism", Tolyatti State University, Tolyatti, \\ Russia \\ ${ }^{2}$ Department of "Physical Education", Tolyatti State University, Tolyatti, Russia
}

\begin{abstract}
The main goal of the work was to study the influence of group fitness training on the physical health of women aged 35-40 years. In the experimental method, functional training, additional equipment, as well as step platforms, exercise bikes and training devices were used. In the course of our work, we studied the dynamics of women's physical health, determined changes in the main stereotypes of lifestyle, nutrition and motor mode. The experimental technique is an effective tool for correcting body weight, contributing to changes in the indicators of total body weight and girth values, influenced the change in the skin and fat folds in the anatomical areas of women. The desire of a person to strengthen his health is his natural need, as a result of physical exercises, there is an increase in the efficiency of the cardiorespiratory system, blood pressure is normalized, the functional state of the musculoskeletal and bone systems improves, efficiency increases, the digestive system improves, and resistance to stress increases. The results of the study allow us to recommend this theoretical and practical material for instructors of group fitness training in clubs of various physical culture and sports orientation.
\end{abstract}

\section{Introduction}

The desire of a person to strengthen his health is his natural need. As Aminova O. S. and other authors note in their works: "Health is a complex and at the same time integral multidimensional dynamic state that develops in the process of realizing the genetic potential in a specific social and ecological environment, allowing a person to exercise his biological and social functions to a greater or lesser extent" [1].

People strive to develop, to learn new things at the same time, developing and improving the intellect, a person gradually loses the motor initiative. In addition, chemical and biological pollution of air and water leads to changes in the immune status of the population and the emergence of new forms of infectious diseases. Health is largely determined by genetics, but it also depends on the following factors:

1) factors that reduce the level of health:

- physical inactivity (lack of optimal physical activity, leading leading to merenrannasta the entire body);

* Corresponding author: schankina@tltsu.ru 
- past illnesses;

- poor nutrition (as the lack of it, and its abundance);

- the adverse conditions of life and work;

- harmful addictions (nicotine, alcohol, drugs);

- frequent stressful stresses;

2) health-promoting factors:

- regular exercise classes;

- tempering (training of the thermoregulatory apparatus);

- compliance with hygiene standards.

Thus, solving the issues of preserving and promoting health, it is necessary, first, to create the most natural conditions for the functioning of the human body, and secondly, by activating the mechanisms of adaptation, to increase the adaptive capabilities of the body. Shilko V. G. and other authors draw attention to the fact that: "A special role in increasing the adaptive capabilities of the human body to stressful influences belongs to physical exercise. Now there is no doubt about the need and importance of adequate physical activity to improve the indicators of psychophysical and somatic health" [2].

A fitness class, like any health-related activity, is held in the form of a group class. This means, first of all, the conduct of the training process by a qualified instructor, who ensures the solution of health problems, as well as the productivity of the lesson. Obviously, when each instructor prepares programs with a wellness orientation, it is necessary to take into account the physiological changes in the body of students that occur during the lesson. Only with a rational distribution of physical exercises can an optimal indicator of the level of health, growth and stability of results be achieved.

The shape type is inherited in most cases. The question arises: whether the type of behavioral reactions of a person aimed at maintaining and maintaining the optimal shape of the body (figure) is inherited. If the inherited type of figure satisfies the person, then the emotional stress associated with its preservation is minimized. In the opposite case, a person constantly has to make certain efforts to combat the shortcomings of the figure, usually with extra pounds. For example, human activity aimed at maintaining optimal physical fitness is largely determined by the characteristics of the individual. A person who is collected, disciplined, and has a strong type of nervous system, it is much easier to maintain his physical form, because he is used to setting certain tasks for himself and certainly achieving their solutions. A person who wants to correct the shortcomings of his figure often asks himself the following questions: why do I not succeed or do it with difficulty, and then all the results achieved are reduced to zero? What is the reason for such phenomena: unwillingness or inability to organize your diet, physical activity? Some begin to blame themselves, their actions, their heredity, etc.

\section{Materials and methods}

The pedagogical research was conducted on the basis of the sports club "Arsenal Fighting" in Tolyatti. Two groups were formed: one control group of 12 women, the other experimental group of 12 women aged 35-40 years. The experimental method included functional training and training with the use of training devices-2 times a week, training women on cardio machines and strength training- 1 time a week. The control group was engaged in group fitness training without the use of exercise equipment and cardio equipment.

In the content of classes with women of the experimental group, strength exercises performed on block simulators with a given amplitude, free weights were used, they occupied $36.4 \%$ (20 minutes) of the lesson. The aerobic part accounted for $54.5 \%$ of the 
total time and was performed with a load of $80 \%$ heart rate $(\max )$, i.e. $137 \pm 2$ beats $/ \mathrm{min}$ (moderate zone).

\section{Results and discussion}

Nazarenko L. D., Timoshina I. N., Mingalisheva I. A. note in their works: "One of the widespread types of physical culture and sports activities is fitness aerobics, the popularity of which is constantly growing due to its accessibility, entertainment, emotionality; positive impact on the psychoemotional, volitional, motivational spheres; focus on versatile and harmonious development, improving one's own image" [3].

Ryabchikov A. I. draws attention to the fact that: "The boundaries of the term fitness are still not precisely defined and vague, and the content is very, very extensive. Let's define the scope of this term:

- a variety of types and means-includes not only various types of aerobics, but also swimming, running for health, athletic gymnastics on modern simulators, etc.;

- the absence of a competitive moment in the form of tournaments, which does not exclude a competitive method in the course of training activities" [4].

Women's group fitness training sessions favorably affect the adaptive processes of the cardiovascular system (in terms of heart rate) and increase the level of physical performance with a decrease in total body weight. Kosheleva M. V. and Ponomareva N. I., after analyzing the impact of the training process of various types of aerobics on the morphofunctional indicators of the body of middle-aged women, write: "Physical activity has a positive effect on the psychophysical sphere of those involved. Given the popularity of fitness training among population groups, it is necessary to create a scientific basis for determining the impact of physical activity on people's health" [5].

According to the body mass index of women, you can determine the degree of deviation of body weight from normal to overweight or underweight. As a result of the exercises, changes were noted that indicate the effectiveness of functional training and training with the use of simulators, in the experimental group, the total body weight index changed by $8.2 \%$, higher in relation to the values in the control group of $3.3 \%$.

Table 1. Women's Body mass index.

\begin{tabular}{|c|c|c|c|}
\hline Group & $\begin{array}{c}\text { Before the } \\
\text { experiment }\end{array}$ & $\begin{array}{c}\text { After the } \\
\text { experiment }\end{array}$ & $\begin{array}{c}\text { Changes } \\
\text { \% }\end{array}$ \\
\hline Control & $27.5 \pm 7.0$ & $26.6 \pm 5.4$ & 3.3 \\
\hline Experimental & $26.7 \pm 6.3$ & $24.5 \pm 4.8$ & 8.2 \\
\hline
\end{tabular}

It should be noted that the experimental set of exercises is an effective tool for correcting body weight, contributing to changes in the indicators of total body weight and girth values, and had a great impact on the change in the skin and fat folds in the anatomical zones: the abdomen on the side, the hip inside, the shoulder behind. The relative index of the fat component in the experimental group of women decreased by $26.3 \%$, which is an average of $12.8 \mathrm{~kg}$ per group. Classes with women require constant monitoring, making appropriate adjustments to the content of classes, methods of teaching the technique of physical exercises, in the parameters of the volume, intensity and duration of physical activity. The formation of motor skills in fitness is based on the regular use of motor actions.

Kharlampenkova Yu. A. writes: "Speaking of conducting group classes, in our opinion, it is necessary to include strength exercises in the main part of the class, which will increase not only the functional capabilities of the body of women, but also more effectively affect the processes of fat burning. In power aerobics classes, we recommend increasing the time 
of the aerobic part and using additional equipment and weights to increase the intensity of the load" [6].

Mingalisheva I. A., Nazarenko L. D., Timoshina I. N. claim that: "The concept of fitness popularity is based on ensuring versatile development, harmonization of physiological functions, increasing general and motor culture, intellectual and spiritual and moral development, physical improvement, and the effectiveness of competitive activities" [7].

The variety of means and their repeated repetition contribute to the formation of the necessary motor skills, the stability of which often depends on the achievements of the students. An increase in the level of physical fitness, respectively, contributes to an increase in the level of health. For the development of physical qualities of women, both general means of physical training and special means are used. Music is a rhythmic stimulus and stimulates the physiological processes of the body in the motor and vegetative zones of the central nervous system, disinhibits the motor centers, gives cheerfulness, adjusts the psyche, improves well-being. Rhythmic music in fitness classes improves mood and emotionality.

Tashmanova N. V., studying the influence of music accompaniment of fitness classes on the development of coordination abilities, writes: "In fitness aerobics, music is used as a leader, i.e. it sets the rhythm, character and controls the pace of movements. The musical rhythm organizes the movements, improves the mood of the students. Positive emotions cause the desire to perform movements more energetically, which increases their impact on the body, contributes to improving performance, as well as improving health and active recreation. Music can also be used as a learning factor, as movements are easier to remember" [8].

Nazarenko L. D., Kasatkina N. A., Mingalisheva I. A. also note: "The coach makes the students match the rhythm of movements with the rhythm of breathing, alternating fast and slow pace, wide amplitude. Draws the attention of students to the need to concentrate on the main element of the technique, then its details, connecting movements; unity, emotionality, grace" [9].

In solving the health problems of fitness classes, it is necessary to take into account the physiological characteristics of the female body, as well as age and psychoemotional state. It should be noted that the experimental set of exercises is an effective tool for correcting body weight, contributing to changes in the indicators of total body weight and girth values, and had a great impact on the change in the skin and fat folds in the anatomical zones: the abdomen on the side, the hip inside, the shoulder behind.

Table 2. Changes in indicators of skin and fat folds $(\mathrm{cm}$.).

\begin{tabular}{|l|c|c|c|c|c|}
\hline \multirow{2}{*}{ Indicators } & \multicolumn{2}{|c|}{ KG } & \multicolumn{2}{c|}{ EG } & \multirow{2}{*}{ P } \\
\cline { 2 - 5 } & Before & After & Before & After & \\
\hline Back of the shoulder, cm & $2.6 \pm 0.2$ & $2.8 \pm 0.6$ & $2.4 \pm 0.3$ & $1.9 \pm 0.2$ & $\leq 0.05$ \\
\hline Top of the back, cm & $2.3 \pm 0.3$ & $2.2 \pm 0.4$ & $2.2 \pm 0.2$ & $1.6 \pm 0.2$ & $\leq 0.05$ \\
\hline Lower back, cm & $2.4 \pm 0.3$ & $2.0 \pm 0.2$ & $2.4 \pm 0.3$ & $1.8 \pm 0.2$ & $\leq 0.05$ \\
\hline Belly on the side, cm & $2.4 \pm 0.3$ & $1.8 \pm 0.3$ & $2.3 \pm 0.3$ & $1.5 \pm 0.2$ & $\leq 0.05$ \\
\hline Inner thigh, cm & $2.7 \pm 0.3$ & $1.9 \pm 0.3$ & $2.8 \pm 0.4$ & $1.9 \pm 0.3$ & $\leq 0.05$ \\
\hline
\end{tabular}

Some women who are overweight experience a constant state of discomfort, and therefore their behavior looks somewhat neurotic, agitated. A person's dissatisfaction with their appearance can manifest itself in a number of other situations: fear of public speaking, problems in relations with the opposite sex, etc. Intense anxiety that accompanies women in such situations is one of the most painful affects, a manifestation of long-term emotional stress. For example, a woman knows that it would not hurt her to lose weight, to put herself in the appropriate shape, but tries not to think about it, postpones the process for the future. 
If a person does not manage to keep himself in good physical shape, then he begins to avoid critical comments from friends and others, sometimes comes into conflict with them, closes himself, surrounds himself, either with people with similar problems, or indifferent to his problems. If a fat person has an anxiety that he will not be able to lose weight, lose extra pounds and look good, then his actions on this occasion will be uncertain, inconsistent. At the same time, a person may be aware that anxiety does not allow him to successfully cope with this task. Anxiety associated with weight loss activities will offset the pleasure that a person could get in the event of parting with extra pounds. But if a person experiences a slight degree of anxiety, which is quite natural, then it will give additional interest and excitement to his actions. If a woman is very worried about her appearance, then she does not need to despair, but should deliberately approach the solution of this problem: make a daily routine, determine the amount of physical activity and the nature of nutrition, arm yourself with theoretical knowledge on this problem, etc. The categories of women who consider staying in a fitness club as a kind of way of spending leisure time were identified. For them, the results do not matter much and much less affect the attitude towards themselves. The study of psychophysiological indicators of the body of women engaged in fitness allows us to determine the effectiveness of the training process, its impact on physical performance, health, anxiety and the state of the psyche as a whole. According to some scientists, extroverted women are more sociable, impulsive, flexible in behavior, more initiative, adapt faster to the conditions of training, but show less perseverance when performing exercises. Many of them are more difficult to observe the necessary diet, they are less collected. Women-introverts, on the contrary, are less sociable, differ in isolation, some passivity of actions, but quite a lot of perseverance. They constantly analyze their behavior, lifestyle, this category of women is more difficult to adapt to the conditions of a fitness center. In the stressful conditions of muscular activity, it was much more difficult for these women to adapt, to learn how to perform the necessary exercises correctly

According to the results of the survey in the course of the pedagogical experiment, $50 \%$ of women improved their health and received positive emotions, $42 \%$ of women achieved figure correction, $38 \%$ of women relieved stress and fatigue.

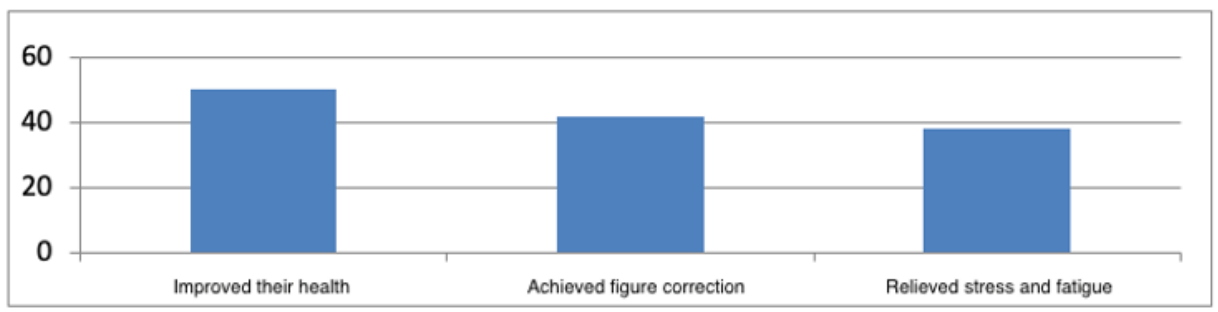

Fig. 1. Results of the women's survey.

The most active group of women who attend classes in fitness clubs are representatives of the first half of adulthood (up to 40 years). The limiting factor is the lack of free time and the high price of fitness services. In the fitness industry, there is a constant process of developing and implementing new areas of training, i.e. updating the content of classes by combining exercises without objects and with different equipment and inventory, the volume and intensity of physical activity. But the main result of a separate activity or system is the achievement of a health-improving effect while maintaining, maintaining and improving the level of physical health against the background of changes in the functional state of the body of women. 
In the process of training activity, the following principles are generally implemented: consciousness and activity, consistency, clarity, scientific, adequacy or individualization of the load, strength, repetition, consistency, gradualness, etc.

The principle of consciousness and activity. It is based on the socio-psychological laws of the individual's activity, i.e. not without taking into account individual and social needs.

The principle of consistency. It is based on the continuity of tasks, tools and methods of training within a single lesson and a series of lessons. The beginning of the implementation of this principle is planning.

The principle of clarity involves the organization of object-sensory and verbal-logical understanding of the studied motor action. It also motivates women engaged in active work to solve a specific task.

The principle of science encourages the coach and the student to build a training process that meets the current levels of scientific knowledge.

The strength principle makes it possible to evaluate the final result, which shows the determination of the women involved in the relevant activities.

The principle of adequacy or individualization of the load provides for mandatory compliance with the available measure of difficulties according to the capabilities of the students (state of health, age, gender, individual mental and physical characteristics, level of fitness). The individual approach of the instructor in the selection of the load involves taking into account the age, level of physical fitness, individual characteristics, including deviations in the state of health.

The principle of repetition provides for the effect of step-by-step formation of motor skills and special knowledge with the help of numerous repetitions.

The principle of consistency is based on a pattern that reflects the subordination of the dependence of the learning effect on the level of completeness of the content of the training process. The principle of gradualness is characterized by the requirement of a gradual increase in the load and is aimed at a constant increase in the requirements for women who are engaged, which consist in a continuous update and complication of the exercises used. With regular systematic loads, a long-term adaptation of the body is formed, but this is provided that the load is constantly increasing. Otherwise, if the load remains unchanged, then the positive effect of adaptation of the body will not be. Of course, we are talking about the growth of the load not from class to class, but over a certain period of time. Applying this principle means gradually increasing the intensity, duration, or frequency of training sessions.

The effectiveness of group fitness training sessions is largely determined by the expediency of physical activity, individual abilities of the individual, only with a rational distribution of physical exercises and load can an optimal level of health, growth and stability of the results of women engaged in it be achieved.

During the pedagogical experiment, we carried out a control test: pulling from hang low lying on the bar $90 \mathrm{~cm}$, flexion and extension of hands in the emphasis lying on the floor, bending forward from a standing position on a gym bench, long jump from the place, lifting the trunk from the supine position (the results are presented in table 3 ). 
Table 3. Indicators of physical health of women engaged in the pedagogical experiment.

\begin{tabular}{|l|c|c|c|}
\hline \multicolumn{1}{|c|}{ Exercise } & KG & EG & P \\
\hline $\begin{array}{l}\text { Pull - up from the vise lying on a low crossbar 90 cm } \\
\text { (number of times) }\end{array}$ & $10.0 \pm 2.0$ & $14.0 \pm 2.0$ & $\leq 0.05$ \\
\hline $\begin{array}{l}\text { Flexion and extension of the arms in the stop lying on the } \\
\text { floor (number of times) }\end{array}$ & $11.0 \pm 2.0$ & $13.0 \pm 3.0$ & $\leq 0.05$ \\
\hline $\begin{array}{l}\text { Forward tilt from the standing position on the gymnastic } \\
\text { bench (from the bench level - cm) }\end{array}$ & $9.0 \pm 2.0$ & $13.0 \pm 2.0$ & $\leq 0.05$ \\
\hline Long jump from a standing position (cm) & $150.0 \pm 4.0$ & $165.0 \pm 3.0$ & $\leq 0.05$ \\
\hline $\begin{array}{l}\text { Lifting the torso from the supine position (number of times } \\
\text { in 1 min) }\end{array}$ & $29.0 \pm 4.0$ & $33.0 \pm 2.0$ & $\leq 0.05$ \\
\hline
\end{tabular}

The women of the experimental group showed better results than the women of the control group, this is due to the fact that the experimental group used functional training, additional equipment, as well as step platforms, exercise bikes and training devices that contributed to the development of physical fitness of the women involved. The control group was engaged only in group fitness training without the use of exercise equipment and cardio equipment.

Grigoriev P. A., Semenova G. I. note in their works: "More and more people who are engaged in fitness or just active motor activity feel pain in their body due to incorrect entry into motor activity or incorrectly selected biomechanics of movement, for which the body is not ready at the moment. The question of what method to choose for improving the quality of movement and, as a result, reducing the overall injury rate for people who lead an active lifestyle remains debatable" [10]. The training should always start with a warm-up, it has a variety of variations of 10-12 minutes, then the next set of different exercises depends on the goals and objectives for the training, at the end of the training, a hitch is performed. During the lesson, the instructor monitors the external signs of overwork of the women involved. In cases of these signs, it reduces physical activity. For women who have missed classes, the physical load in the first classes is reduced, and then gradually brought to the level of normal load. In the structure of the lesson, the physical load gradually increases. The highest level of it is reached by the middle of the main part of the lesson. The correct distribution of physical activity in the parts of the class is controlled by the physiological curve, determined by the pulse rate. This point is especially important, since the healthimproving effect obtained from training with general strengthening exercises can be provided only if there are sufficient reserve forces of the body of those involved. Overloading of the organs and systems involved in physical exercises exhausts the strength and fatigue occurs. In turn, this leads to a decrease in the tone of the neuromuscular apparatus.

Regular use of physical exercises suspends the processes of recurrent changes, allows you to improve your health and slow down the aging of the body. As a result of physical exercise:

1. Increases the efficiency of the cardiorespiratory system:

- reduced the frequency of heart rate (HR);

- blood pressure is normalized;

- increases the vital capacity of the lungs (VEL);

- improves the metabolic processes in the lungs;

- reduces the risk of heart and lung diseases.

2. Improves functional standing the state of the musculoskeletal and bone system:

- there is a physiological hypertrophy of the muscles;

- increases the size and elasticity of blood vessels;

- increases muscle strength and endurance;

- improves the condition of the spine; 
- strengthens the bone tissue.

3. Increases the energy potential of the body:

- improves performance;

- slow down the aging process, the immune system's defensive responses are activated.

4. Improves the functioning of the digestive system:

- optimized oxidative processes;

- regulates the metabolism.

5. Improve the functions of the central nervous system:

- improves the nervous regulation of all body systems, increases resistance to stress.

The majority of women in the experimental and control groups felt positive changes in their health after fitness classes: improved well-being, mood, activity, which indicates the activation of the metabolic process in the body and an increase in vitality. During the entire period of fitness classes, there were no sharp changes in well-being, activity, or mood among them, which indicated their stable mental health, good health, and good adaptation to the conditions of physical exertion. This contributed to a better assimilation and reproduction of physical exercises, as well as a more favorable recovery of the body's energy resources, increased mental and physical performance, which indicates the normalization of the internal emotional state of women.

\section{Conclusions}

Studying the impact of group fitness training on the physical health of women aged 35-40 years, we used functional training, step platforms, exercise bikes, additional equipment and musical accompaniment. As a result of the training, positive changes in the indicators of the functional state of women were noted: the resting pulse (HR p.) decreased by $11.9 \%$ to normal age values, systolic pressure decreased by $9.1 \%$, diastolic-by $8.5 \%$. The relative index of the fat component decreased by $26.3 \%$ in the experimental group of women. In solving health problems, we took into account the physiological characteristics of the female body, as well as age and psychoemotional state. In the control tests: the tightening of visa lying on the low bar $90 \mathrm{~cm}$, flexion and extension of hands in the emphasis lying on the floor, bending forward from the position of standing on a gym bench, long jump from the place, lifting the trunk from the supine position, women in the experimental group showed higher results than women in the control group. This confirms our hypothesis that the use of group fitness training contributes to improving physical health indicators, changing the main lifestyle stereotypes, preventing the preservation and strengthening of the health of women involved.

\section{References}

1. Aminova O. S. et al. Monitoring of health indicators in women of different age groups // Hygiene and Sanitation. - 2019. - Vol. 98. - no. 8. - p. 827-832.

2. Shilko V. G. et al. Changes in the significance of stress factors in female students during fitness classes //Theory and practice of physical culture. - 2018. - no. 10. - p. 57-59.

3. Nazarenko L. D., Timoshina I. N., Mingalisheva I. A. Improving the performing skills of those engaged in fitness aerobics //Theory and practice of physical culture. - 2019. No. 2. - pp. 81-83.

4. Ryabchikov A. I. Fitness and its place in physical culture //Theory and practice of physical culture. - 2017. - No. 5. - pp. 103-104. 
5. Kosheleva M. V., Ponomareva N. I. Analysis of the impact of the training process of various types of aerobics on the morphofunctional indicators of the body of middleaged women //Scientific Notes of the PF Lesgaft University. - 2020. - №. 3 (181).- Pp. 258-263.

6. Kharlamenkova J. A. Accounting somatic features of women of childbearing age in the organization of various sports aerobics / Y. A. Kharlamenkova, N. And. Gardanova // Uchenye Zapiski universiteta im. P. F. Lesgafta. - 2012. - № 3 (85). - P. 184-187.

7. Mingalisheva I. A., Nazarenko L. D., Timoshina I. N. Modeling of improving the performance skills of those engaged in fitness aerobics //Theory and practice of physical culture. - 2018. - No. 2. - pp. 62-64.

8. Tashmanova N. V. Influence of musical accompaniment of fitness classes on the development of students ' coordination abilities //Theory and practice of physical culture. - 2020. - No. 9. - pp. 93-94.

9. Nazarenko L. D., Kasatkina N. A., Mingalisheva I. A. Realization of creative potential in the process of fitness aerobics classes //Theory and practice of physical culture. 2020. - No. 5. - p. 28-30.

10. Grigoriev P. A., Semenova G. I. Functional assessment of movement as a means of reducing injuries in fitness. Sport. Medicine. - 2020. - Vol. 20. - no. 1. - p. 114-122. 